\section{IMAGE UNAVAILABLE FOR COPYRIGHT REASONS}

Will the 'millennium bug' cause microchip mayhem - or is the scare just hype?

to make a lot of money out of saying there is a big problem", says John Crowcroft, professor of network systems at University College London. Crowcroft adds that tests of the core Internet protocols and applications for $\mathrm{y} 2 \mathrm{k}$ problems "found a couple of things where the user might be misled, but otherwise the system functioned fine".

The large scientific databases are also taking a relatively relaxed stance. "I'm not profoundly alarmed," says Graham Cameron, head of services at the European Bioinformatics Institute in Cambridge, England. The institute's systems do not use two-digit dates.

Many UK universities are ignoring the problem, claims one member of the UK government's Year 2000 Task Force. The French atomic energy commission (CEA) has no plans to establish a formal Year 2000 programme, according to a spokeswoman, Corrine Borrell, who says the agency is content with raising awareness among staff.

But scientists who play down the problem are being "extraordinarily irresponsible", asserts Robin Guenier, a consultant who heads the UK task force, and is a former chief executive of the government's Central Computing and Telecommunications Agency.

He says: "This is not being exaggerated. If anything it is being underestimated, and there is little time to fix it." Anthony Parish, director general of the UK Federation of Electronic Industries, agrees: "We think it is a major disaster; it will cost a large amount of money."

One reason for such concerns is growing awareness of the problem of embedded microchips. "These are beginning to be acknowledged as perhaps a bigger problem [than software]," says Simon Reeves, coauthor of a recent book called The Millennium Bomb. Pharmaceutical companies are reported to be highly concerned about the chips used throughout process plants.

But claims that embedded chips will cause widespread problems, such as shutdowns of power plants and aircraft, are contested by several scientists. Darrell Ince, a specialist in critical systems software at the UK Open University, argues that such problems would be localized and rarely cause crashes. "I don't think you will get problems in safety-critical systems such as planes flying upside down."

Declan Butter

\title{
Space Shuttle launch holds key to asteroid mission
}

[WASHINGTON] A controversial US military spacecraft mission to fire a probe into an asteroid's surface may finally get off the ground in 1999 if Congress commits additional funding for next year, and if the National Aeronautics and Space Administration (NASA) offers a free ride on the Space Shuttle.

Managers of the Clementine 2 project hope the Air Force will approve their mission plan as early as next week, which would allow work to resume at the Naval Research Laboratory in Washington DC and at the Air Force Phillips Laboratory in New Mexico. The project has been in abeyance for much of the past 18 months due to budget politics at the Pentagon (see Nature 381,$8 ; 1996$ ).

One result of that controversy is that Clementine 2 is no longer seen as part of a broad "planetary defence" mission to protect the Earth from rogue asteroids. The rationale was always considered suspect in some sectors of the Pentagon, and so advocates of the project now emphasize its advanced technology.

Like the Clementine 1 mission that mapped the Moon in 1994, the project is primarily a test of miniature spacecraft components, cameras and sensors developed for space-based missile defence. The plan calls for the spacecraft to launch in November 1999 and fly past two asteroids - 1986JK and Toutatis - in 2000. A probe would be sent crashing into each one, stirring up material that could be studied by spectrometers and cameras on the main craft.

NASA's involvement in the project is crucial, to "show that there really are valid and exciting scientific objectives", says Eugene Shoemaker, a retired US Geological Survey planetary scientist who heads an informal team set up by the Clementine 2 project office.

The team met last month in Flagstaff, Arizona, to identify scientific goals not only for the first mission but also for a possible follow-on after the two main encounters. Shoemaker says the spacecraft could go on to meet more asteroids, visit the Moon or be sent into deep space to conduct astrophysical parallax studies that would help to locate massive compact halo objects and gamma-ray bursts.

Shoemaker's team will meet again later this month, then report to NASA by 1 June. If the space agency decides to participate, it would establish a permanent science team, handle all research data returned by the spacecraft, and perhaps contribute an instrument in addition to a suite of cameras and spectrometers already under development at the Lawrence Livermore National Laboratory in California.

A second key question is whether NASA will agree to launch Clementine 2 on the Space Shuttle at little or no cost. The shuttle programme chief, Stephen Oswald, said recently in congressional testimony that NASA is interested in providing a launch, but no details have been worked out. Part of the problem, say Clementine supporters, is that until now NASA has been unsure that the Air Force is fully behind the project. Approval of the Clementine 2 mission plan, and the resumption of work at the Naval Research Laboratory and Phillips, should help to ease those doubts.

The final ingredient necessary for Clementine 2 to fly is more money from Congress. The project received only $\$ 45 \mathrm{mil}$ lion last year - half the amount it requested - as the result of a political deal between Republicans and Democrats to trim the defence budget. Without an additional $\$ 50$ million in 1998, the asteroid fly-bys are out of the question, and the project would be scaled back just to test the probes in Earth orbit. Clementine supporters are optimistic that the Senate Armed Services Committee, which authorizes defence spending, will add the money, and that appropriations committees will follow suit.

Clementine is not the only science project being negotiated between NASA and the Air Force. The two agencies are close to finalizing an agreement whereby the Jet Propulsion Laboratory would provide upgraded cameras to three space-tracking telescopes used by the military. The Air Force would gain from the added camera performance, and the space agency would be able to speed up and improve its search for near-Earth asteroids.

Tony Reichhardt

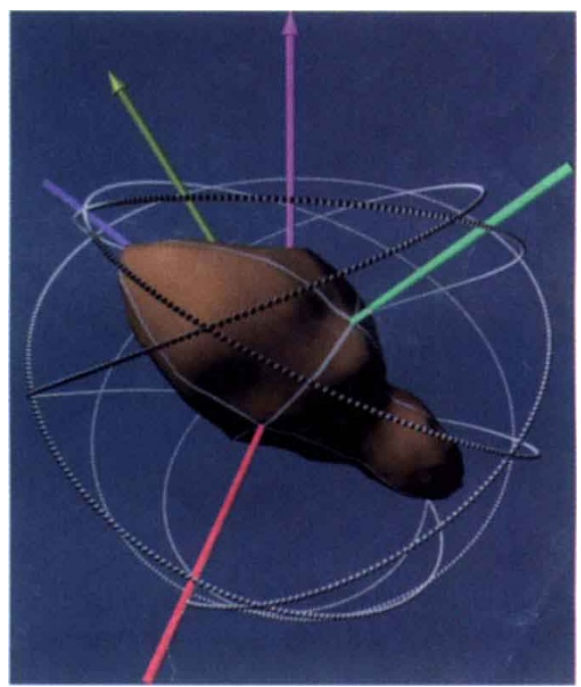

In a spin: Clementine 2 will fire a probe into the Toutatis asteroid (above) if it can hitch a ride on the Space Shuttle. 